\title{
The amorphous solid dispersion of the poorly soluble ABT-I02 forms nano/microparticulate structures in aqueous medium: impact on solubility
}

\author{
This article was published in the following Dove Press journal: \\ International Journal of Nanomedicine \\ 9 November 2012 \\ Number of times this article has been viewed
}

\author{
Kerstin J Frank ${ }^{1,3}$ \\ Ulrich Westedt ${ }^{2}$ \\ Karin M Rosenblatt ${ }^{2}$ \\ Peter Hölig ${ }^{2}$ \\ Jörg Rosenberg² \\ Markus Mägerlein ${ }^{2}$ \\ Gert Fricker ${ }^{3}$ \\ Martin Brandl ${ }^{1}$ \\ 'Department of Physics, Chemistry \\ and Pharmacy, University of Southern \\ Denmark, Odense, Denmark; \\ ${ }^{2} \mathrm{Abbott} \mathrm{GmbH}$ and Co. KG, \\ Ludwigshafen, Germany; ${ }^{3}$ Department \\ of Pharmaceutical Technology, \\ University of Heidelberg, Heidelberg, \\ Germany
}

\begin{abstract}
Amorphous solid dispersions (ASDs) are a promising formulation approach for poorly soluble active pharmaceutical ingredients (APIs), because they ideally enhance both dissolution rate and solubility. However, the mechanism behind this is not understood in detail. In the present study, we investigated the supramolecular and the nano/microparticulate structures that emerge spontaneously upon dispersion of an ASD in aqueous medium and elucidated their influence on solubility. The ASD, prepared by hot melt extrusion, contained the poorly soluble ABT-102 (solubility in buffer, $0.05 \mu \mathrm{g} / \mathrm{mL}$ ), a hydrophilic polymer, and three surfactants. The apparent solubility of ABT-102 from the ASD-formulation was enhanced up to 200 times in comparison to crystalline ABT-102. At the same time, the molecular solubility, as assessed by inverse equilibrium dialysis, was enhanced two times. Asymmetrical flow field-flow fractionation in combination with a multiangle light-scattering detector, an ultraviolet detector, and a refractometer enabled us to separate and identify the various supramolecular assemblies that were present in the aqueous dispersions of the API-free ASD (placebo) and of binary/ternary blends of the ingredients. Thus, the supramolecular assemblies with a molar mass between 20,000 and 90,000 could be assigned to the polyvinylpyrrolidone/vinyl acetate 64 , while two other kinds of assemblies were assigned to different surfactant assemblies (micelles). The amount of ABT-102 remaining associated with each of the assemblies upon fractionation was quantified offline with high-performance liquid chromatography-ultraviolet-visible. The polymeric and the micellar fraction contributed to the substantial increase in apparent solubility of ABT-102. Furthermore, a microparticulate fraction was isolated by centrifugation and analyzed by scanning electron microscopy, X-ray scattering, and infrared spectroscopy. The microparticles were found to be amorphous and to contain two of the surfactants besides ABT-102 as the main component. The amorphous microparticles are assumed to be the origin of the observed increase in molecular solubility ("true" supersaturation).
\end{abstract}

Keywords: solid dispersion, AFIFFF, micelles, solubility, supersaturation

\section{Introduction}

Over $50 \%$ of newly developed active pharmaceutical ingredients (APIs) comprise poor solubility but high permeability properties. Oral bioavailability of these substances is thus restricted by their low solubility and/or slow dissolution rate. This behavior has become a major challenge for formulation scientists over the last few years. Various strategies have been used to enhance solubility and/or dissolution rate of oral formulations (for a recent review see Singh et $\mathrm{al}^{1}$ ).
Department of Physics, Chemistry and Pharmacy, University of Southern

Denmark, Campusvej 55, 5230

Odense C, Denmark

Tel +4565502525

Fax +4566158780

Email mmb@ifk.sdu.dk 
One popular approach is the use of solubilizing formulations, which increase the apparent solubility of the API by inclusion within micelles (micellar solutions). Recent studies, however, indicate that enhanced apparent solubility via micellar solubilization does not necessarily enhance permeability and bioavailability. ${ }^{2,3}$

Another approach - amorphous solid dispersions (ASDs) formed by melt extrusion - has become more and more popular over the last few years. ${ }^{4-6}$ ASDs are either solid solutions or solid dispersions of amorphous API in a polymer matrix. This state of the API might lead to an accelerated dissolution rate and enhanced apparent solubility, sometimes called supersaturation. Strictly speaking, in supersaturated solutions, the drug should be molecularly dissolved.7 "True" supersaturation is a thermodynamically unstable state, but may be stabilized to provide an increased concentration of molecularly dissolved API over time (parachute) ${ }^{8}$ Various polymers as well as surfactants are used as precipitation inhibitors, the latter also termed wetting agents or solubilizers. ${ }^{9-11}$ These excipients might form different kinds of colloidal structures upon dispersion in aqueous media, which are assumed to impact the apparent solubility. In the present study, we elucidate the mechanism behind the increased solubility of the poorly soluble ABT102 in dispersions of an ASD in comparison to crystalline ABT-102. ABT-102 is a transient receptor potential vanilloid type 1 (TRPV1) antagonist. The TRPV1 receptor is a nexus in pain transmission and is therefore regarded as a promising target in the treatment against pain. The pharmacology is described in detail by Kym et al. ${ }^{12}$ ABT- 102 has a high $\log \mathrm{P}$ (5.28) and is poorly soluble in aqueous medium $(0.05 \mu \mathrm{g} /$ $\mathrm{mL}$ in buffer). ${ }^{12}$ The aqueous dispersion of an ASD containing the poorly soluble ABT-102, a hydrophilic polymer, and three surfactants was investigated in terms of which kinds of assemblies are present in the colloidal fraction. This rather complex ASD was chosen because it appeared promising in terms of in vivo bioavailability (unpublished data). The study was performed with asymmetrical flow field-flow fractionation (AFIFFF) coupled to a multiangle light-scattering (MALS) detector, an ultraviolet (UV) detector and a refractive index (RI) detector. In addition, the microparticles, which are too big to be fractionated by AFIFFF, were separated by centrifugation and analyzed by scanning electron microscopy (SEM), powder X-ray scattering and Fourier-transform infrared (FT-IR) spectroscopy.

This experimental setup made it possible to get a deeper understanding of the macromolecules between $10 \mathrm{kDa}$ and $100 \mathrm{kDa}$ and of the nano- and microparticulate structures from $10 \mathrm{~nm}$ up to $100 \mu \mathrm{m}$, which emerge upon dispersion of the extrudate in buffer.

\section{Materials and methods}

Crystalline ABT-102 (99.4\% purity), as well as the extrudates were provided by Abbott GmbH \& Co. KG (Ludwigshafen, Germany). The excipients polysorbate 80 , sucrose palmitate, polyvinylpyrrolidone/vinyl acetate 64 (PVP/VA 64) and poloxamer 188 were also kindly provided by Abbott (the purity of the excipients was according to the specifications in the European Pharmacopeia). Sodium nitrate and acetonitrile (analytical grade) were obtained from Sigma-Aldrich Chemie (Munich, Germany).

\section{Manufacturing of melt extrudates}

\section{Material preparation and mixing}

Prior to extrusion, solid and liquid excipients were mixed using a granulator (model P10; Diosna, Osnabrueck, Germany) (rotor speed, $400 \mathrm{rpm}$; chopper, level 2 for 3 minutes). Subsequently, the excipient mixture was blended twice with the API in a container blender (type Bohle PM400) at $6 \mathrm{rpm}$. Between each blending step, the mixture was sieved through a 1-mm screen (Comil type U5; Quadro, Ontario, Canada) in order to ensure a homogeneous distribution of the API.

\section{Hot melt extrusion}

Melt extrusion was performed using a Micro 18 co-rotating, intermeshing twin-screw extruder (Leistritz, Nuremberg, Germany). The screw diameter was $18 \mathrm{~mm}$ at a length-todiameter ratio of 30 . The barrel segment adjacent to the powder feeder was water-cooled. The second barrel had a temperature of $80^{\circ} \mathrm{C}$. All other barrel segments were heated to $107^{\circ} \mathrm{C}$. Vacuum was applied to remove residual moisture during the extrusion process. Screw speed and feeding rate were kept constant at $130 \mathrm{rpm}$ and $2.0 \mathrm{~kg} /$ hour, respectively. The physical mixture was fed with a loss-in-weight feeder system (type KT 20; K-Tron, Pitman, USA).

\section{Sample preparation for analysis}

Dispersions of the melt extrudates were prepared by dispersing the melt extrudates (beads) in the dispersion medium (sodium nitrate $10 \mathrm{mM}$ ) in a volumetric flask and allowing it to stir for 1 hour at $37^{\circ} \mathrm{C}$ at $400 \mathrm{rpm}$. For the experiments in this study, the melt extrudates were dispersed in concentrations of $3.2 \mathrm{mg}$ (extrudate) $/ \mathrm{mL}$ (ABT-102-containing extrudate) and $3.02 \mathrm{mg}$ (extrudate) $/ \mathrm{mL}$ (placebo extrudate). Solutions of each single excipient were prepared by dissolution of the corresponding amount of excipient in the $10 \mathrm{mM}$ 
sodium nitrate solution. Mixtures of the single excipients were prepared by mixing their solutions.

\section{Analysis of apparent and molecular solubility}

The dispersions of the ASDs and of crystalline ABT-102 were prepared as described in the previous section. Afterwards, a defined volume of the aqueous dispersions was transferred into centrifugation tubes and centrifuged for 60 minutes at $18,500 \mathrm{~g}$ at $37^{\circ} \mathrm{C}$ (J2-MC; Beckman Coulter, Brea, CA). After centrifugation, aliquots of the supernatant were withdrawn, immediately diluted with acetonitrile, and analyzed with high-performance liquid chromatography-UV-visible (HPLC-UV/Vis), as described in the following section. The inverse equilibrium dialysis for determination of molecular solubility was performed as described by Frank et al. ${ }^{3}$ First, the sample dispersions were put into beakers (donor). Thereafter, dialysis vials (cutoff, $3.5 \mathrm{kDa}$; Gene Bioapplication, Yavne, Israel), were filled with blank buffer (acceptor). The dialysis vials were put into the sponge-like floating devices and set into the beakers. Aliquots were taken from inside the dialysis vials as soon as the equilibrium was reached (20 hours). The aliquots were diluted with acetonitrile and the amount of ABT-102 quantified with HPLC-UV/Vis.

\section{Quantification of ABT-I02 by HPLC-UV/Vis}

The instrument consisted of a separation unit (Ultimate 3000; Dionex, Sunnyvale, CA) with a C18 column $(4.6 \times 300 \mathrm{~mm})$ coupled to a UV/Vis detector (Ultimate 3000$)$. Measurements were performed at a flow rate of $1.5 \mathrm{~mL} /$ minute with a gradient, starting with $45 \%$ eluent $\mathrm{A}(0.1 \%$ trifluoroacetic acid in water) and $55 \%$ eluent B ( $0.1 \%$ trifluoroacetic acid in acetonitrile), shifting to $20 \%$ eluent $\mathrm{A}$ and $80 \%$ eluent $\mathrm{B}$ over 10 minutes, followed by 3 minutes of isocratic flow profile. The detection wavelength of ABT-102 was $\lambda=267 \mathrm{~nm}$ and the retention time of ABT-102 was $8.2 \mathrm{~min}$. The injection volume was $100 \mu \mathrm{L}$.

\section{Analysis of the colloidal structures Asymmetrical flow field-flow fractionation}

For size separation, AFIFF (Eclipse 3+; Wyatt Technology, Dernbach, Germany) was used. The separation channel was coupled online to a UV/Vis detector (Agilent Technologies, Boeblingen, Germany), a MALS detector (DAWN EOS) and a refractometer (Optilab rEX), both from Wyatt Technology.

During the separation step, the detector flow was $1.0 \mathrm{~mL} /$ minute and the cross flow started with $1.5 \mathrm{~mL} /$ minute as a gradient that ended after 20 minutes at a cross-flow rate of $0.1 \mathrm{~mL} /$ minute. The gradient was followed by a constant cross flow of $0.1 \mathrm{~mL} /$ minute for 20 minutes.

A $10-\mathrm{kDa}$ regenerated cellulose membrane and a 250 $\mu \mathrm{m}$ spacer were used. Data were evaluated with ASTRA 5.3.2.1 5 software (Wyatt Technology). The Zimm model was used for size calculations. For the calculation of the molar mass of the polymer, an differential refractive index of $\mathrm{dn} / \mathrm{dc}=0.15 \mathrm{~mL} / \mathrm{g}$ was used.

Each sequence of a sample (set of more than three runs of one sample solution/dispersion) was repeated at least twice (three different days) with freshly prepared sample solutions/dispersions. In Figures 1-4, the fractograms of one representative run are shown.

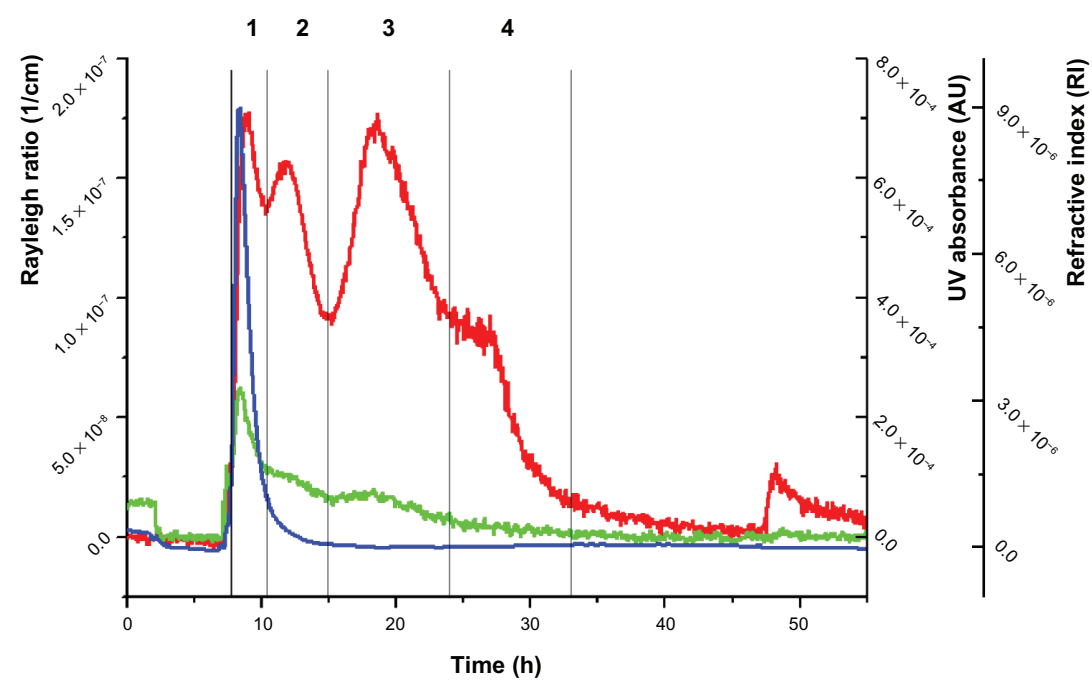

Figure I Fractogram of the placebo extrudate.

Notes: Red line: Rayleigh ratio $(\mathrm{I} / \mathrm{cm})$ (light-scattering signal at angle $90^{\circ}$ ); green line, UV/Vis signal; blue line, differential refractive index. 


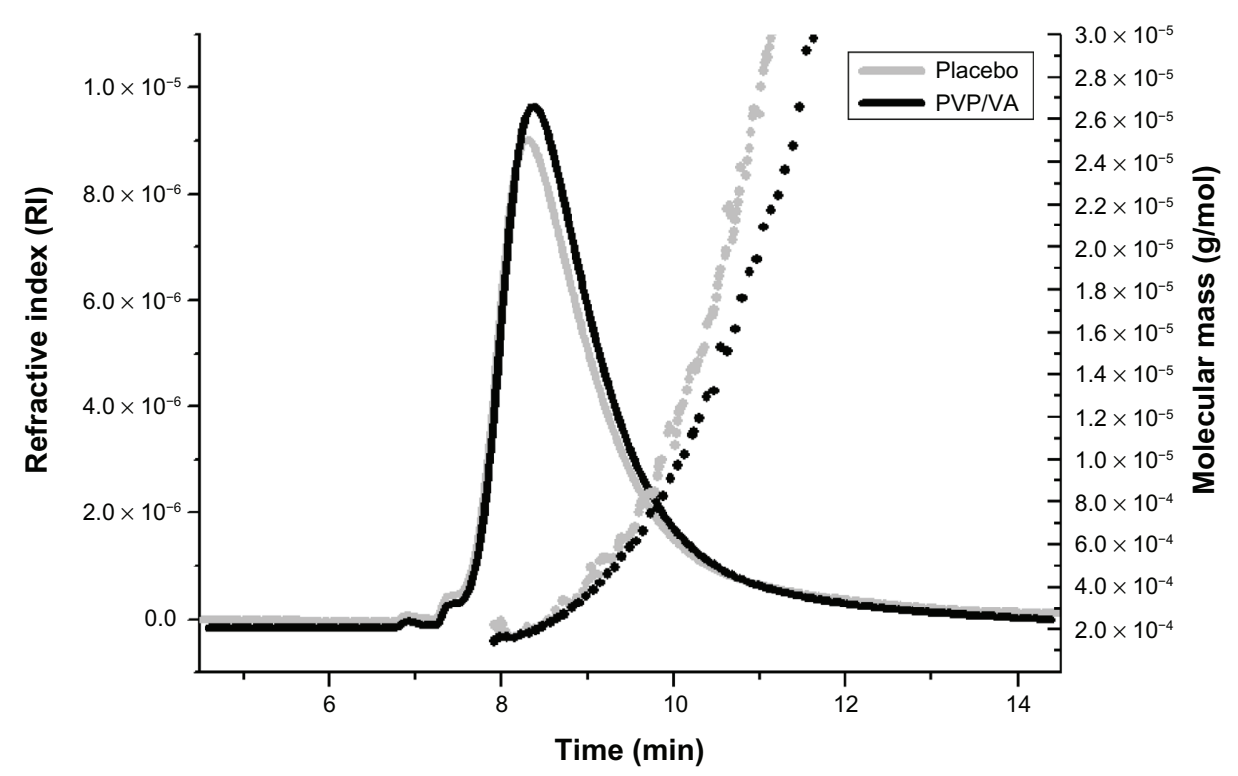

Figure 2 Comparison of the placebo extrudate (black) with PVP/VA 64.

Notes: Solid line, refractive index; dotted line, molecular mass.

\section{Quantification of ABT-102 in the fractions}

The fractions after separation with AFIFFF were collected in centrifugation tubes. Then the water was removed by freezedrying and the dry precipitate was dissolved in a 1:1 mixture of water and acetonitrile. Afterwards, aliquots were analyzed with HPLC-UV/Vis.

\section{Analysis of the precipitate}

\section{$X$-ray scattering}

The ABT-102-containing microparticles were investigated for crystalline parts of ABT-102 using powder X-ray diffraction.
Diffraction patterns were recorded using an X'Pert Pro MPD diffractometer (Panalytical, Einshoven, Netherlands) with a pixel detector, data collector, and HighScore software. Measurements were performed with a $\mathrm{Cu} \mathrm{K} \alpha$ radiation source at $40 \mathrm{kV}$ voltage and $40 \mathrm{~mA}$ current from $2.5^{\circ}$ to $3.2^{\circ} 2$-theta in a continuous scanning mode. The instrument was set to a step width of $0.006^{\circ}$ 2-theta and a measurement time per step of 3000 seconds. The irradiated sample length was $20 \mathrm{~mm}$.

A 29-mm diameter powder-diffraction sample holder with a zero-background holder made from single crystal

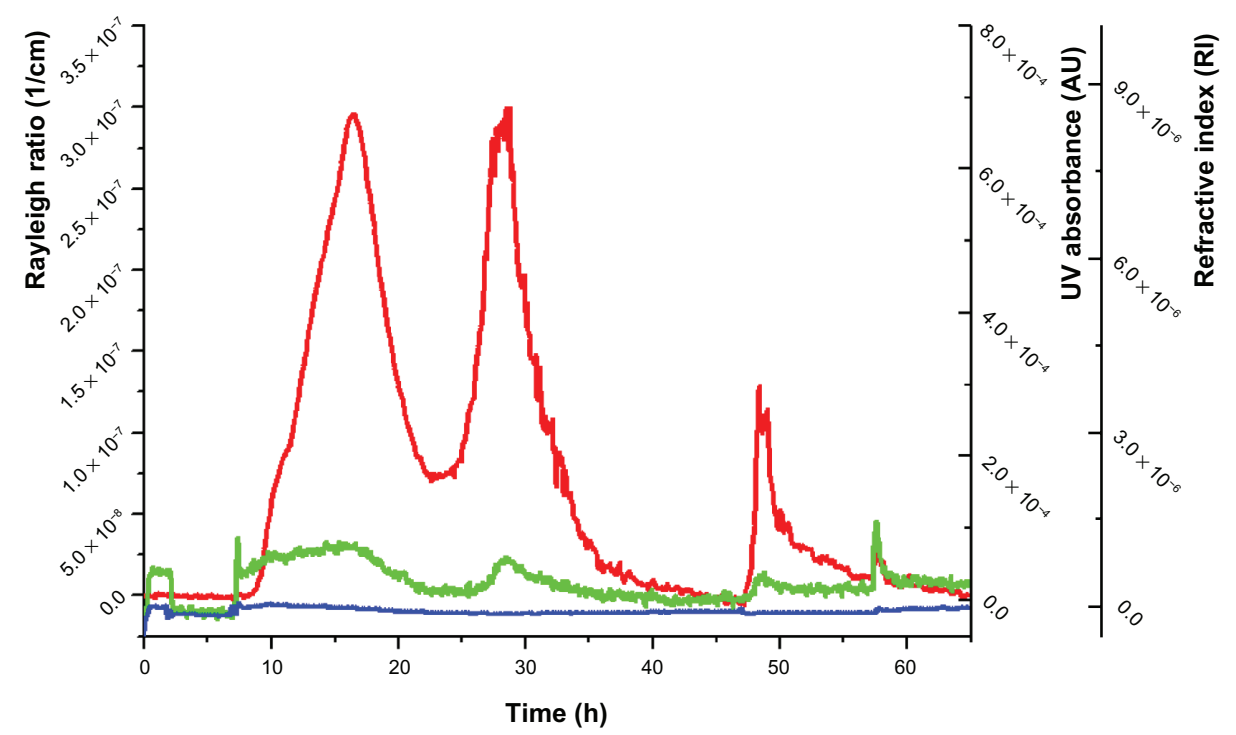

Figure 3 Fractogram of the mixture of sucrose palmitate and polysorbate 80 .

Notes: Red line, Rayleigh ratio $(1 / \mathrm{cm})$ (light-scattering signal at angle $90^{\circ}$ ); green line, UV/Vis signal; blue line, differential refractive index. 


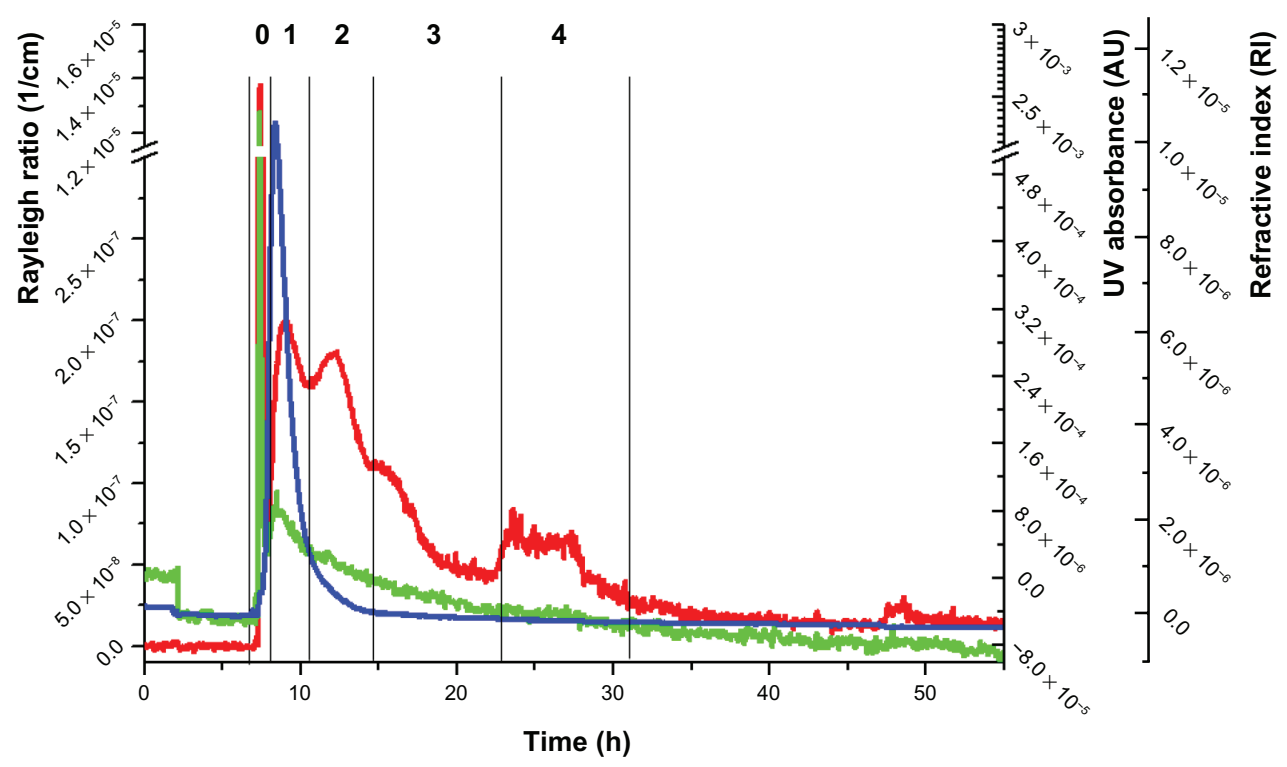

Figure 4 Fractogram of the ABT-102-containing extrudate.

Notes: Red line, Rayleigh ratio $(1 / \mathrm{cm})$ (light-scattering signal at angle $\left.90^{\circ}\right)$; green line, UV/Vis signal; blue line, differential refractive index.

silicon (Panalytical) was used for the measurements and the sample was covered with a polyimide (Kapton) film (Chemplex, Palm City, FL).

\section{Infrared spectroscopy}

The powder samples were analyzed with an FT-IR spectrometer (Spectrum One, Waltham, MA). The resolution was $4 \mathrm{~cm}^{-1}$ and the samples were analyzed in five measuring cycles.

\section{Scanning electron microscopy}

The dry-powder samples were attached to the sample holder with double-sided tape and coated with a $200-\AA ̊$ gold layer using an ion sputter coater (JFC-1100; Jeol, Tokyo, Japan). Then they were observed with an SEM (LEO 435 VP; Zeiss, Oberkochen, Germany).

\section{Results}

\section{Molecular solubility and apparent solubility}

The molecular solubility was assessed by inverse equilibrium dialysis (cutoff, $3.5 \mathrm{kDa}$ ). The concentration of molecularly dissolved ABT-102 was $0.15 \mu \mathrm{g} / \mathrm{mL} \pm 0.01 \mu \mathrm{g} / \mathrm{mL}$ for the dispersions of the ASD in $10 \mathrm{mM} \mathrm{NaNO}_{3}$ (mean \pm standard deviation $[\mathrm{SD}] ; \mathrm{n}=7$ ). This was twice the concentration of molecularly dissolved ABT-102 in the case of crystalline ABT-102 (molecular structure and physical and chemical properties, Table 1), dispersed in buffer $(0.08 \mu \mathrm{g} / \mathrm{mL} \pm 0.01 \mu \mathrm{g} / \mathrm{mL} ;$ mean $\pm \mathrm{SD} ; \mathrm{n}=6)$. An increased concentration of molecularly dissolved ABT-102 for the ASD indicates that there emerged "true" supersaturation. The apparent solubility (assessed by benchtop centrifugation) was $9.74 \mu \mathrm{g} / \mathrm{mL} \pm 1.76 \mu \mathrm{g} / \mathrm{mL}(\mathrm{n}=11)$. The high apparent solubility is assumed to be caused by a solubilization of ABT-102 by micelles and/or polymeric structures. To investigate if and which supramolecular assemblies emerge upon dispersion of the extrudate, AFIFFF-MALS/UV/RI studies were performed.

\section{AFIFFF analysis of an aqueous dispersion of the placebo extrudate}

First, the aqueous dispersion of the placebo extrudate (composition in Table 2) was examined. When fractionating the dispersion of the placebo extrudate, the MALS signal in the fractogram revealed four partially overlapping peaks at elution times from 7 to 35 minutes (peaks 1-4, Figure 1).

The UV detector indicated one distinct peak (peak 1) followed by a broad shoulder. The RI detector showed just one peak (peak 1). One should mention that the washout phase starts at 48 minutes and that the peaks afterwards are thus regarded as artifacts. The apparent molar mass or root mean-square radii were calculated for each of the peaks (Table 3).

In order to get an idea, if any of the four peaks may be assigned to one of the excipients, aqueous solutions of PVP/VA 64 , poloxamer 188 , polysorbate 80 , and sucrose palmitate (molecular structures and some physical and chemical properties; Table 1) were individually 
Table I Molecular structures and physical and chemical properties of the ingredients

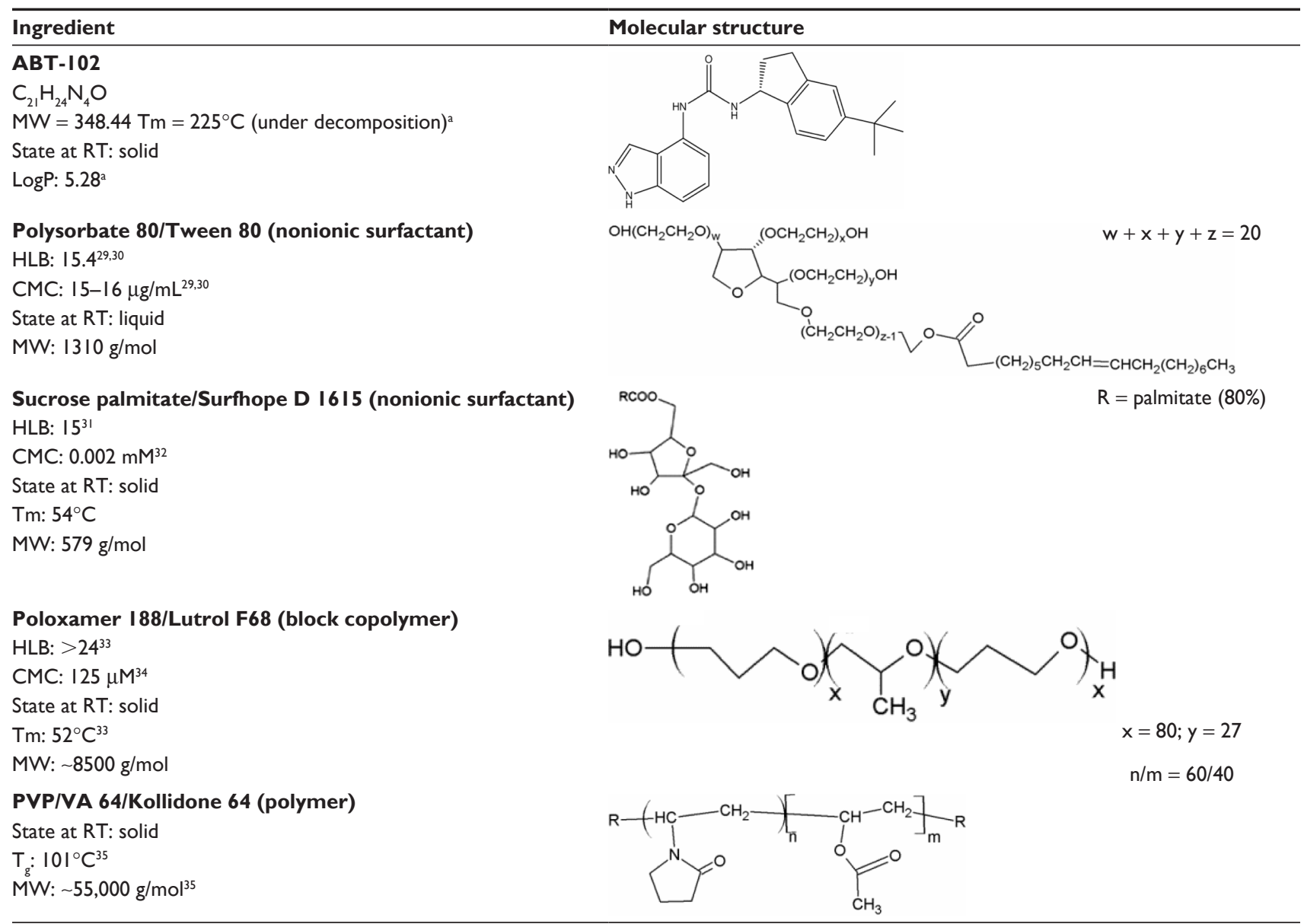

Note: ${ }^{a}$ Abbott data.

Abbreviations: CMC, critical micelle concentration; HLB, hydrophilic/lipophilic balance; MW, molecular weight; RT, room temperature; $T_{g}$, glass transition temperature; Tm, melting point.

fractionated under the same conditions. The resulting fractograms were compared to the fractogram of the placebo extrudate. Among all four single components, only the PVP/VA 64 fractogram showed similarities to one peak of the placebo-fractogram (Figure 2) (the other fractograms of the single components are not shown). In the next step,

Table 2 Composition of the extrudates

\begin{tabular}{|c|c|c|}
\hline Ingredients & $\begin{array}{l}\text { ABT-containing } \\
\text { extrudate percentage }\end{array}$ & $\begin{array}{l}\text { Placebo } \\
\text { percentage }\end{array}$ \\
\hline ABT-102 & 5 & 0 \\
\hline $\begin{array}{l}\text { Copovidon Typ K28 } \\
\text { (Kollidon VA 64) }\end{array}$ & 81.5 & 85.7 \\
\hline $\begin{array}{l}\text { Sucrose palmitate } \\
\text { (Surfhope D-1615) }\end{array}$ & 1.5 & 1.6 \\
\hline $\begin{array}{l}\text { Poloxamer I88 } \\
\text { (Pluronic F68) }\end{array}$ & 6.0 & 6.3 \\
\hline $\begin{array}{l}\text { Polysorbate } 80 \\
\text { (Tween } 80 \text { ) }\end{array}$ & 5.0 & 5.3 \\
\hline $\begin{array}{l}\text { Fumed silica } \\
\text { (Aerosil 200) }\end{array}$ & 1.0 & I.I \\
\hline
\end{tabular}

binary mixtures of the solutions of the excipients were analyzed. In most cases, the binary mixtures did not show any peaks at elution times above 15 minutes (data not shown). Sucrose palmitate mixed with polysorbate 80 , in contrast, revealed two distinct peaks, one starting at 9 minutes and the other starting at 23 minutes (Figure 3). This mixture was the only binary mixture that revealed signals with an elution time above 15 minutes.

In order to identify the contribution of each of the four ingredients to the fractogram of the whole mixture and to evaluate the ability of the single compounds to form complex assemblies, ternary blends of their aqueous solutions were analyzed. The influence of the missing ingredient was evaluated by comparing the resulting fractograms with the fractogram of the placebo extrudate. The observations are summarized in Table 4. When comparing the fractogram of the dispersions of the placebo extrudate with the solutions of the excipients, it has to be noted that the melt extrudate is expected to contain an even distribution of the surfactant 
Table 3 Placebo extrudate: peaks and sizes

\begin{tabular}{|c|c|c|c|c|c|c|}
\hline Peak number & Retention time (minutes) & Light-scattering signal & UV signal & RI signal & Rms radius (nm) & Molar mass (g/mol) \\
\hline I & $7.7-11.0$ & Yes & Yes & Yes & - & $20,000-90,000$ \\
\hline 2 & $11.0-15.0$ & Yes & - & - & I5-30 (weak signal) & - \\
\hline 3 & $15.0-23.0$ & Yes & - & - & $30-60$ & - \\
\hline 4 & $23.0-33.0$ & Yes & - & - & $>70$ & - \\
\hline
\end{tabular}

Abbreviations: RI, refractive index; Rms, root mean square; UV, ultraviolet.

molecules and the polymer due to the melt extrusion process. This could lead to slightly different assemblies in comparison to a mixture of the aqueous solutions of the surfactants and the polymer.

\section{Analysis of an aqueous dispersion of the ABT-102-containing extrudate}

The fractogram of the aqueous dispersion of the ABT-102containing formulation is presented in Figure 4. The main difference in comparison to the placebo extrudate was a sharp peak at 8 minutes, designated as peak 0 , which was detected by the MALS detector and the UV detector.

AFIFFF theory implies that smaller macromolecules or particles elute ahead of bigger particles. This would mean that the size of the assemblies in peak 0 should be between $10 \mathrm{kDa}$ and $20 \mathrm{kDa}$. Alternatively, the observed peak may be due to steric elution, meaning that large particles elute as soon as the elution step starts, because they are too big to be fractionated at the given channel height, defined by the thickness of the chosen spacer $(250 \mu \mathrm{m})$. Another modification in comparison to the placebo extrudate was seen in the lightscattering signals of peaks 3 and 4 . There were two signals seen in the fractogram of the ABT-102-containing extrudate: one with an elution time from 14 minutes to 23 minutes and the other with an elution time from 23 minutes to 31.5 minutes. The elution times were in the same range as those of peaks 3 and 4 of the placebo extrudate. However, the peak shape was different and the areas under the curve (AUCs) were decreased, especially that of peak 3, which was the predominant one in the fractogram of the placebo extrudate.
To check whether peak 0 was due to rather large particles, the dispersion was centrifuged for 60 minutes at $18,500 \mathrm{~g}$ prior to the AFIFFF study, and the clear supernatant obtained this way was investigated. It was seen that peak 0 was drastically decreased (data not shown).

In the next step, the distribution of ABT-102 over the different supramolecular assemblies in fractions $0-4$ of the centrifuged sample (only supernatant) was evaluated. These assemblies are assumed to cause the up-to-200-fold increased apparent solubility $(9.74 \pm 1.76 \mu \mathrm{g} / \mathrm{mL} ; \mathrm{n}=11)$ that was observed for the dispersion of the ASD in $10 \mathrm{mM} \mathrm{NaNO}_{3}$ in comparison to the crystalline ABT-102. The results of the collection and offline quantification of ABT-102 of the fractions $0-4$ in the supernatant after fractionation with AFIFFF are presented in Figure 5. Most of the ABT-102 was found in fraction 1 , followed by fractions 3 and 4 . The total amount, which was detected in all AFIFFF fractions (0-4), was $0.821 \pm 0.138 \mu \mathrm{g}$ $(n=5$, mean $\pm S D)$. This represents about $56 \%$ of the injected amount (injection volume, $150 \mu \mathrm{L}$ of the supernatant).

\section{Analysis of the precipitate}

In the previous section, the distribution of ABT-102 over the different nanoparticulate structures in the supernatant after centrifugation was described. In this section, we focus on the larger particles that were spun down during the centrifugation. The precipitate, which contained about $90 \%$ of the total amount of the ABT-102 in the aqueous dispersion of the ASD, was analyzed in detail. SEM of the freeze-dried precipitate showed particles in the size range $1-100 \mu \mathrm{m}$. The appearance of these particles was different from the ABT-102 crystals, as demonstrated in Figure 6. Powder X-ray-scattering analysis of

Table 4 Overview of the peaks in the ternary mixtures

\begin{tabular}{lllll}
\hline $\begin{array}{l}\text { Placebo peak } \\
\text { number }\end{array}$ & Mix without & Mix without & Mix without sucrose & Mix without \\
\hline PVP/VA 64 & poloxamer 188 & palmitate & polysorbate 80 \\
2 & - & + & + & + \\
3 & + & + & + & Partial \\
4 & + & + & Partial & - \\
\hline
\end{tabular}

Notes: +, peak seen in the fractogram of the ternary mixtures; -, no peak detected at this elution time. 


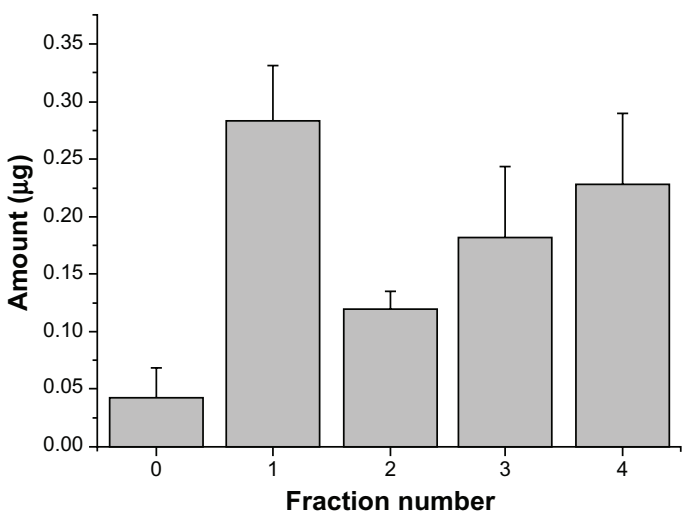

Figure 5 Distribution of ABT- 102 over the fractions of peaks $0-4$ of the centrifuged sample.

Note: The total injected amount was $1.46 \mu \mathrm{g} \pm 0.26 \mu \mathrm{g}$ (calculated from apparent solubility data).

the wet precipitate indicated the absence of ABT-102 crystallites within the limits of the method (Figure 7). The crystalline ABT102 revealed a peak at the position $2.98^{\circ} 2$ Theta. In the case of the microparticles, there was no peak observed at this position. The angle range was chosen for the indication of crystallinity of ABT-102, because a previous study showed that crystalline ABT-102 showed its biggest reflex in that range. ${ }^{13}$

FT-IR studies of the dried precipitate were done to determine the ingredients of the amorphous microparticles. Figure 8 presents the spectra of the single compounds and of the precipitate (microparticles). The relevant bands in the spectra are marked and further described in Table 5. It is clearly seen that the ABT-102 was present in the precipitate, because of its characteristic bands $1,5,6$, and 8 . The characteristic band of the PVP/VA 64 (number 4) is not present in the spectra of the precipitate. Polysorbate 80 and/or sucrose palmitate are assumed to be present in the precipitate, as band number 3, which is seen in their spectra, is represented in the spectra of the precipitate as well.

\section{Discussion}

The release of a poorly soluble drug from a hydrophilic polymer matrix has been under investigation for a long time. There were

A

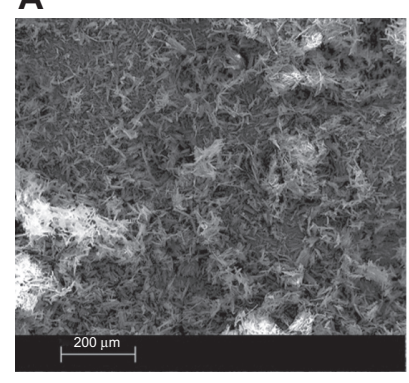

Figure 6 SEM images of crystalline ABT-102 (A) and the freeze dried precipitate (B). elaborate hypotheses about the mechanism published quite some years ago. ${ }^{14,15}$ However the mechanism is still far from well understood. It is generally accepted that a kinetically stable ASD is desirable, ${ }^{16}$ ie, recrystallization of the API within the glassy matrix is expected to bring about inferior drug-release properties. In consequence, stabilization of the amorphous state such as molecular interactions and mobility has been in focus. ${ }^{17}$ Karavas et al compared two types of binary solid dispersions (polymer/API). ${ }^{18}$ They observed amorphous or crystalline API particles as a function of drug-polymer interactions. These particles appeared to persist upon dispersion in aqueous medium. Albers et al described that for a given binary formulation consisting of a polymer and an API, melt extrusion conditions were decisive for recrystallization tendency. ${ }^{19}$ In case of opaque solid dispersions, a tendency of drug recrystallization in contact with aqueous medium was reported. In clear solid solutions, a release of drug in a polymer-controlled manner was seen. Warren et al summarized the potential action of hydrophilic polymers as precipitation inhibitors. ${ }^{9}$

Ternary or higher-grade amorphous solid dispersions containing one or several surfactants besides the API and the hydrophilic polymer are even more complex. Several studies have been performed to evaluate the role of the surfactants in the solid state. They can be used to decrease the glass transition temperature of the blend (eg, Ghebremeskel et $\mathrm{al}^{20}$ ). In contrast, only few studies have looked into the role of surfactants on the release mechanism. Tho et al reported formation of nano- and microparticles in the aqueous dispersions of surfactant-containing melt extrudates. ${ }^{21}$ Kanzer et al ${ }^{22}$ demonstrated that there may coexist several types of supramolecular or nanoparticulate structures in aqueous dispersions of API-containing melt extrudates. However, the apparent solubility or ratio of supersaturation was not evaluated in their study at the same time.

The ASD investigated here was able to increase the molecular solubility ("true" supersaturation) and to increase apparent solubility in comparison to crystalline ABT-102. Molecular solubility in our understanding refers to single ABT-102 molecules surrounded by a hydration shell. Apparent solubility additionally takes into account the colloidal solubilized ABT-102. Potential colloidal structures may arise from the polymer and/or surfactants contained in the formulation. Kanzer at al suggested AFIFFF as a promising analytical tool for micelles and other nanoparticulate assemblies. ${ }^{22}$

To evaluate the nature and composition of the colloidal structures in this study, the AFIFFF fractogram of the placebo extrudate was compared with the fractograms of single compounds and binary and ternary mixtures of the single compounds. 


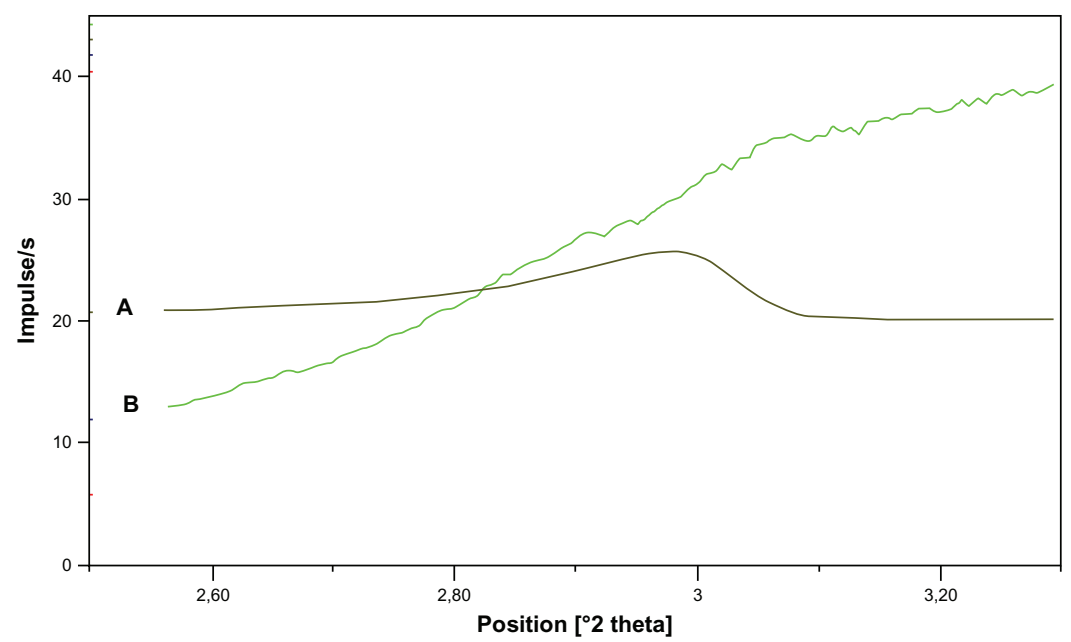

Figure 7 (A and B) Powder X-ray diffractogram of the precipitate and of crystalline ABT-102. (A) (Brown line): crystalline ABT- 102; (B) (green line): precipitate. Note: The diffractogram of crystalline ABT- 102 was downscaled two times by factor 0.0125 .

Peak 1 is assumed to be composed mainly of PVP/VA 64. The light-scattering signal, the RI peak, and the UV/Vis peak, as well as the molar mass of the PVP/VA 64 single compound are in good correlation with peak 1 in the fractogram of the placebo extrudate. Additionally, a quantification of the amount of ABT-102 in the fractions of the centrifuged sample revealed that the fraction corresponding to peak 1 contained the highest amount of ABT-102 among all fractions collected from AFIFFF. The relatively poor recovery and high standard variations in the amount of ABT-102 in the fractions can be explained by the huge surface area, to which ABT-102 might adsorb. Furthermore, some of the ABT-102, which was bound to the polymer and/or other supramolecular assemblies, might have been "washed off" from the colloidal structures during the run. The bottom part of the separation channel is comprised of a semipermeable membrane with a cutoff of $10 \mathrm{kDa}$. Thus, molecularly dissolved ABT-102 is assumed to disappear with the cross flow through the membrane and cannot be detected in the detector flow.

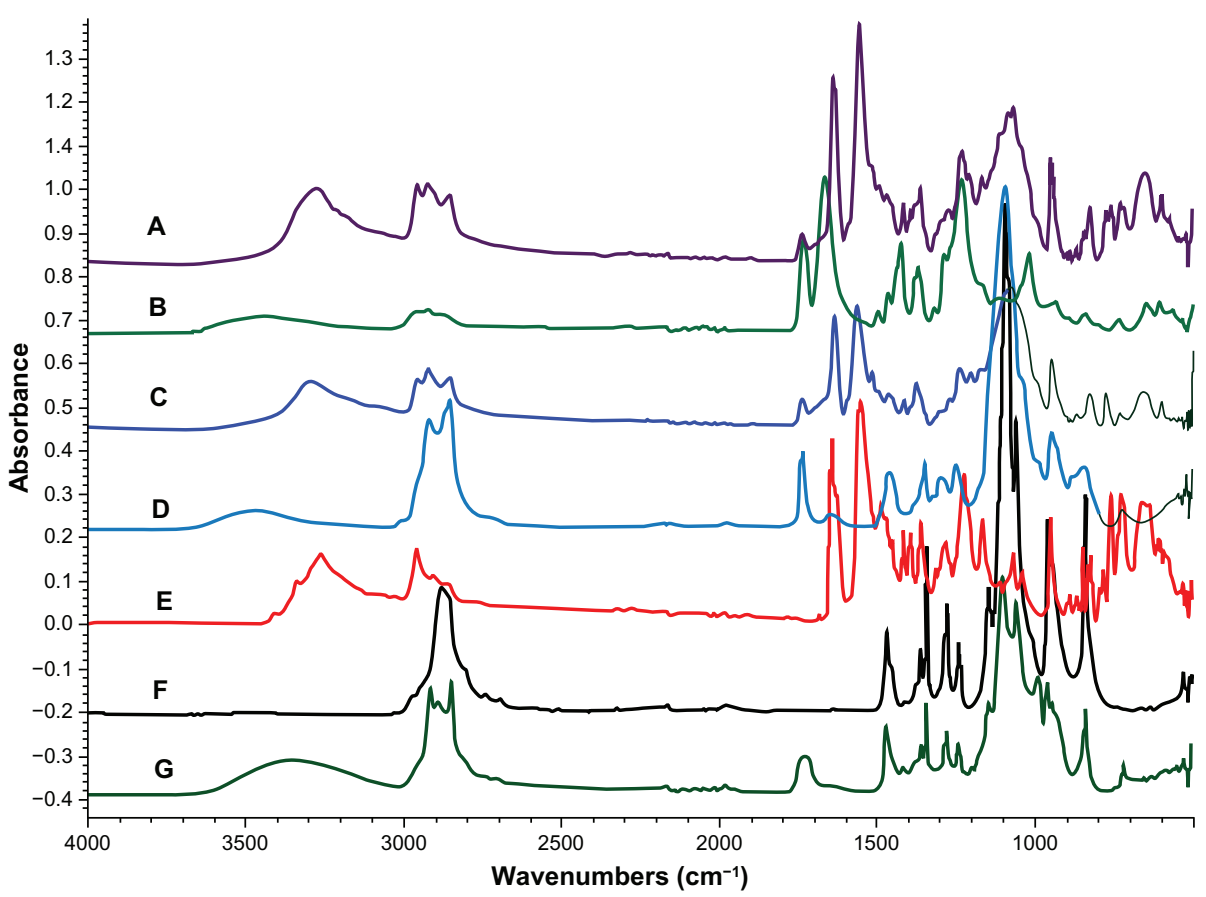

Figure 8 (A-G) FT-IR spectra of the dried precipitate (from a dispersion in water and in buffer), and for comparison, the spectra of ABT-I02 (crystalline) and the excipients. (A) (Violet): precipitate in water; (C) (blue): precipitate in buffer; (B) (green): PVP/VA 64; (D) (light blue): polysorbate 80; (E) (red): ABT-I02; (F) (black): poloxamer I88; (G) (dark green): sucrose palmitate. 
Table 5 Overview of the bands in the FT-IR spectra in Figure 8

\begin{tabular}{llll}
\hline Band number & Wavenumbers $\left(\mathbf{c m}^{-1}\right)$ & Characteristic absorption & Band seen in the spectrum of \\
\hline $\mathrm{I}$ & 3300 & $\mathrm{~N}-\mathrm{H}$ stretch & Precipitate, ABT-I02 \\
2 & $3000-2800$ & $\mathrm{C}-\mathrm{H}$ aliphatic stretch & All spectra \\
3 & 1750 & $\mathrm{C}=\mathrm{O}$ stretch aliphatic ester & Precipitate, PVP/VA 64, polysorbate 80, sucrose palmitate \\
4 & 1660 & $\mathrm{C}=\mathrm{O}$ stretch lactame & PVP/VA 64 \\
5 & $1625-1575$ & $\mathrm{C}-\mathrm{C}$ stretch aromatic & Precipitate, ABT-I02 \\
6 & $\mathrm{C}-\mathrm{O}-\mathrm{C}$ stretch aliphatic & Precipitate, polysorbate 80, poloxamer I88, sucrose palmitate \\
7 & $900-650$ & $\mathrm{C}-\mathrm{H}$ aromatic out of plane bend & Precipitate, ABT-I02 \\
\hline
\end{tabular}

It was not possible to determine one excipient or a mixture of excipients that could be assigned to peak 2 , as none of the single compounds or mixtures revealed a peak at exactly that position. The light-scattering signal of the PVP/VA 64 solution (peak 1) showed a small shoulder at this position, and the fractogram of the binary mixture of sucrose palmitate and polysorbate 80 (peaks 3 and 4) revealed a small shoulder at that elution time, too. Consequently, we assume that peak 2 might be caused by an overlapping of peak 1 and 3 .

Peak 3 was generated only in the presence of polysorbate 80. In addition, the peak occurred to be smaller in the absence of sucrose palmitate. This leads to the conclusion that there are structures formed that contain a mixture of these two ingredients. We assume that it might be mixed micelles, but as far as we know there are no micelles of that kind described in the literature yet. The analysis of the mixture of the solutions of polysorbate 80 and sucrose palmitate proved that there emerged two kinds of supramolecular assemblies in this mix. Peak 4 is supposed to consist of a mixture of polysorbate 80 and sucrose palmitate, too. The two micellar peaks with different sizes might be attributed to two different mixedmicelle types. It is has been described in the literature that the generation of two different kinds of micelles is possible in mixed micellar systems..$^{23,24}$ In this case, both micellar peaks (3 and 4) contained a distinct amount of ABT-102. From this point of view, there was no relevant difference observed between these two kinds of mixed micelles.

The smaller AUC of the micellar light-scattering signal, which was observed for the ABT-102-containing extrudate (peaks 3 and 4) in comparison to the placebo extrudate, is assumed to be caused by a loss of surfactants due to precipitation of the surfactants (polysorbate 80 and sucrose palmitate) with ABT-102 into microparticles.

Poloxamer 188 did not play a role in the generation of mixed assemblies, probably because of its block-copolymeric properties, which decrease the possibility to form mixed supramolecular assemblies (micelles) with the other two surfactants due to steric hindrance.

To conclude, two categories of colloidal structures have been detected: a polymeric one and the micellar one, which both contained ABT-102. The observations here were performed at a concentration of the extrudate that was well above the critical micellar concentration of the surfactants (Table 1) and of the placebo extrudate (data not shown). In the case of other concentrations, the structures that emerge might be different. One has to bear in mind that AFlFFF, as most fractionation methods, implies a major dilution of the sample during focusing and fractionation, concomitant with a change in ionic strength. The supramolecular assemblies that were found are thus not necessarily identical with those present in the sample prior to fractionation.

It has been reported that polymers are able to form hydrogen-bound stabilized complexes with APIs and prevent it therefore from nucleation. ${ }^{25}$ Another theory is that the polymers bind to the surface of crystallites or nuclei and hinder further crystal growth. ${ }^{26,27}$

In this study, the complex of the polymer and the API in the polymeric fraction (peak 1) was rather stable, because there was still ABT-102 bound to the polymer after 12 minutes runtime and dilution with approximately $15 \mathrm{~mL}$ of buffer. The molecular mass distribution of the polymeric peak in the dispersion of the extrudate was in good accordance with the molecular mass distribution of the polymer solution. We assume that the interactions of the polymer and ABT-102 took place on a molecular level, because otherwise (binding of the polymer to nanoparticles of the API) the molecular mass of the polymeric fraction would have been increased and the bigger particles would have eluted later.

Furthermore, the amorphous microparticles in the precipitate are assumed to be not covered with PVP/VA 64, because all PVP/VA 64 was in solution, which is concluded from the AUC of the concentration-dependent RI signal of peak 1. In addition, FT-IR analysis of the precipitate revealed that there was no PVP/VA 64 present in the precipitate.

The ABT-102-containing extrudate revealed a decreased AUC of the micellar peaks (peaks 3 and 4) in comparison to the placebo extrudate. This reduced signal is attributed to a loss of polysorbate 80 and/or sucrose palmitate. FT-IR analysis demonstrated that the precipitate did contain polysorbate $80 \mathrm{and}$ /or sucrose palmitate. It might be possible that the 
surfactants acted as crystallization inhibitors and stabilized the amorphous state of the microparticles. Recent studies on the effect of surfactants on the growth of crystals in supersaturated solutions by Overhoff et $\mathrm{al}^{10}$ and Brewster et $\mathrm{al}^{28}$ are in accordance with our results. Overhoff et $\mathrm{al}^{10}$ hypothesized that the crystal growth of tacrolimus was actively hindered through adsorption of sodium dodecyl sulfate onto the surface of tiny drug particles, which inhibits crystal growth and precipitation. In our case, the surfactant(s) might have bound to the precipitating amorphous microparticles and thereby might have inhibited their crystallization. Further studies are under way, which may elucidate the composition of the precipitate and the intermolecular bindings between the ingredients more precisely. Taken together, we could detect three different categories of ABT-102 in the aqueous dispersion of the ASD. One category was the molecularly dissolved ABT-102, which was found to show enhanced concentration in comparison to the crystalline ABT-102 ("true" supersaturation). In addition, there emerged a colloidal fraction (category 2) that solubilized the ABT-102 by absorption to the polymer and inclusion into nanoparticulate supramolecular assemblies (micelles). This category contributed mainly to the up-to-200-fold-higher apparent solubility (concentration in the supernatant) in comparison to crystalline ABT-102 (Figure 9A). In addition, a microparticulate category was found that contained most of the ABT-102 ( $90 \%)$ that was present

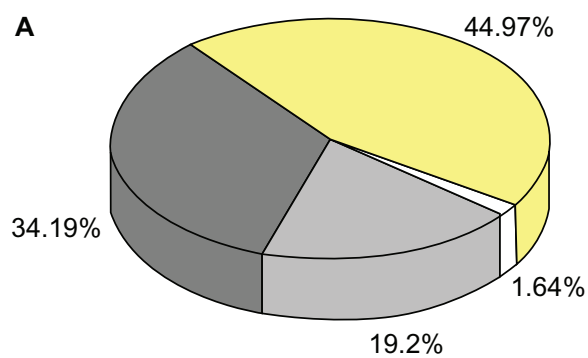

$19.2 \%$

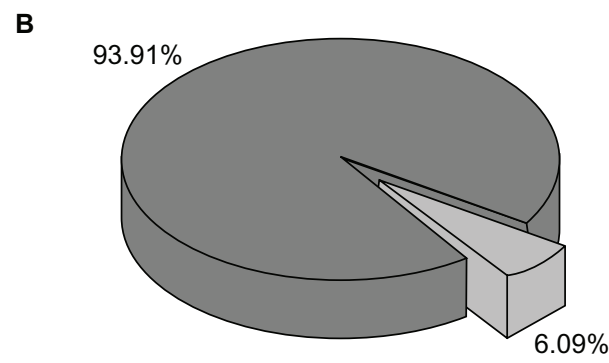

Figure 9 (A) Contribution of various structures to the apparent solubility; $100 \%=9.74 \mu \mathrm{g} / \mathrm{mL}$; white, molecular dissolved ABT-102; light grey, polymericbound; dark grey, micellar-bound; yellow, loss due to poor recovery of the AFIFFF experiment. (B) Distribution of ABT-102 in the complete sample dispersion; $6.09 \%=9.74 \mu \mathrm{g} / \mathrm{mL} ; 100 \%=160 \mu \mathrm{g} / \mathrm{mL}$ (weight in amount); light grey, dissolved fraction; dark grey, microparticulate structures. in the whole aqueous dispersion of the ASD (Figure 9B). The influence of the various structures on the bioavailability of ABT-102 will be evaluated in a further study.

\section{Conclusion}

An ABT-102-containing ASD was found to increase its apparent solubility up to 200 times in comparison to crystalline ABT-102. Furthermore, inverse equilibrium dialysis indicated that the concentration of molecularly dissolved drug was enhanced beyond its solubility limit (two times), indicating "true" supersaturation. The aqueous dispersion of the ASD was screened for supramolecular assemblies in the nanometer range as well as for microparticles. The combination of centrifugation and AFIFFF enables the separation of the complex system into three categories: a polymeric, a micellar, and a microparticulate one. The polymer and the mixed supramolecular assemblies (micelles) of polysorbate 80 and sucrose palmitate acted clearly as solubilizing agents and contributed thereby to the increase of apparent solubility. Further investigations into the microparticles with X-ray scattering, SEM, and FT-IR indicated that the microparticles consisted mainly of amorphous ABT-102. In addition, they contained surfactant(s) that are assumed to stabilize the amorphous state. These microparticles are hypothesized to account for the ability of the ASD to generate supersaturation, ie, an increase of the molecular solubility. This is to our knowledge the first report giving in-depth insight into the supramolecular assemblies and microparticles in the dispersion of an ASD, as well as into their contribution towards enhanced apparent solubility and molecular solubility.

\section{Acknowledgments}

The authors would like to thank Stefanie Heber and David Gessner at Abbott GmbH and Co. KG (Ludwigshafen, Germany) for performing the X-ray scattering and infraredspectroscopy studies. Furthermore, we would like to thank Danny Kyrping for helping with the SEM studies. We would like to thank Abbott $\mathrm{GmbH}$ and Co. KG for financial support of this study.

\section{Disclosure}

The authors report no conflicts of interest in this work.

\section{References}

1. Singh A, Worku ZA, Van den Mooter G. Oral formulation strategies to improve solubility of poorly water-soluble drugs. Expert Opin Drug Deliv. 2011;8(10):1361-1378.

2. Fischer SM, Brandl M, Fricker G. Effect of the non-ionic surfactant Poloxamer 188 on passive permeability of poorly soluble drugs across Caco-2 cell monolayers. Eur J Pharm Biopharm. 2011;79(2): 416-422. 
3. Frank KJ, Westedt U, Rosenblatt KM, et al. Impact of FaSSIF on the solubility and dissolution-/permeation rate of a poorly water-soluble compound. Eur J Pharm Sci. 2012;47(1):16-20.

4. Bikiaris DN. Solid dispersions, part I: recent evolutions and future opportunities in manufacturing methods for dissolution rate enhancement of poorly water-soluble drugs. Expert Opin Drug Deliv. 2011; 8(11):1501-1519.

5. Bikiaris DN. Solid dispersions, part II: new strategies in manufacturing methods for dissolution rate enhancement of poorly water-soluble drugs. Expert Opin Drug Deliv. 2011;8(12):1663-1680.

6. Breitenbach J. Melt extrusion: from process to drug delivery technology. Eur J Pharm Biopharm. 2002;54(2):107-117.

7. Brouwers J, Brewster ME, Augustijns P. Supersaturating drug delivery systems: the answer to solubility-limited oral bioavailability? J Pharm Sci. 2009;98(8):2549-2572.

8. Augustijns P, Brewster ME. Supersaturating drug delivery systems: fast is not necessarily good enough. J Pharm Sci. 2012;101(1):7-9.

9. Warren DB, Benameur H, Porter CJ, Pouton CW. Using polymeric precipitation inhibitors to improve the absorption of poorly water-soluble drugs: a mechanistic basis for utility. J Drug Target. 2010;18(10): 704-731.

10. Overhoff KA, McConville JT, Yang W, Johnston KP, Peters JI, Williams RO 3rd. Effect of stabilizer on the maximum degree and extent of supersaturation and oral absorption of tacrolimus made by ultra-rapid freezing. Pharm Res. 2008;25(1):167-175.

11. Janssens S, Van den Mooter G. Review: physical chemistry of solid dispersions. J Pharm Pharmacol. 2009;61(12):1571-1586.

12. Kym PR, Kort ME, Hutchins CW. Analgesic potential of TRPV1 antagonists. Biochem Pharmacol. 2009;78(3):211-216.

13. Frank KJ, Rosenblatt KM, Westedt U, et al. Amorphous solid dispersion enhances permeation of poorly soluble ABT-102: true supersaturation vs apparent solubility enhancement. Int J Pharm. 2012;437(1-2):288-293.

14. Craig DQ. The mechanisms of drug release from solid dispersions in water-soluble polymers. Int J Pharm. 2002;231(2):131-144.

15. Corrigan OI. Mechanisms of dissolution of fast release solid dispersions. Drug Dev Ind Pharm. 1985;11(2-3):697-724.

16. Swallen SF, Kearns KL, Mapes MK, et al. Organic glasses with exceptional thermodynamic and kinetic stability. Science. 2007;315(5810): 353-356.

17. Yu LX, Ellison CD, Conner DP, Lesko LJ, Hussain AS. Influence of drug release properties of conventional solid dosage forms on the systemic exposure of highly soluble drugs. AAPS Pharm Sci. 2001;3(3):E24.

18. Karavas E, Georgarakis E, Sigalas MP, Avgoustakis K, Bikiaris D. Investigation of the release mechanism of a sparingly water-soluble drug from solid dispersions in hydrophilic carriers based on physical state of drug, particle size distribution and drug-polymer interactions. Eur J Pharm Biopharm. 2007;66(3):334-347.

19. Albers J, Alles R, Matthee K, Knop K, Nahrup JS, Kleinebudde P. Mechanism of drug release from polymethacrylate-based extrudates and milled strands prepared by hot-melt extrusion. Eur J Pharm Biopharm. 2009;71(2):387-394.
20. Ghebremeskel AN, Vemavarapu C, Lodaya M. Use of surfactants as plasticizers in preparing solid dispersions of poorly soluble API: selection of polymer-surfactant combinations using solubility parameters and testing the processability. Int J Pharm. 2007;328(2): 119-129.

21. Tho I, Liepold B, Rosenberg J, Maegerlein M, Brandl M, Fricker G. Formation of nano/micro-dispersions with improved dissolution properties upon dispersion of ritonavir melt extrudate in aqueous media. Eur J Pharm Sci. 2010;40(1):25-32.

22. Kanzer J, Hupfeld S, Vasskog T, et al. In situ formation of nanoparticles upon dispersion of melt extrudate formulations in aqueous medium assessed by asymmetrical flow field-flow fractionation. J Pharm Biomed Anal. 2010;53(3):359-365.

23. Lerk PC, Sucker HH, Eicke HF. Micellization and solubilization behavior of sucrose laurate, a new pharmaceutical excipient. Pharm Dev Technol. 1996;1(1):27-36.

24. Yamamoto M, Ando H, Arima S, Aramaki K. Rheological properties of wormlike micellar solutions being available in wide temperature range in sucrose palmitate systems. J Oleo Sci. 2009;58(6):303-311.

25. Raghavan SL, Kiepfer B, Davis AF, Kazarian SG, Hadgraft J. Membrane transport of hydrocortisone acetate from supersaturated solutions; the role of polymers. Int J Pharm. 2001;221(1-2):95-105.

26. Alonzo DE, Zhang GG, Zhou D, Gao Y, Taylor LS. Understanding the behavior of amorphous pharmaceutical systems during dissolution. Pharm Res. 2010;27(4):608-618.

27. Lindfors L, Forssen S, Westergren J, Olsson U. Nucleation and crystal growth in supersaturated solutions of a model drug. J Colloid Interface. 2008;325(2):404-413.

28. Brewster ME, Vandecruys R, Verreck G, Peeters J. Supersaturating drug delivery systems: effect of hydrophilic cyclodextrins and other excipients on the formation and stabilization of supersaturated drug solutions. Pharmazie. 2008;63(3):217-220.

29. Neugebauer JM. Detergents: an overview. Methods Enzymol. 1990;182:239-253.

30. Dawson RMC, Elliott DC, Elliott WH, Jones KM. Data for Biochemical Research. New York: Oxford University Press; 1989.

31. Mitsubishi-Kagaku Foods Corporation. Surfhope SE Pharma. Available from: http://www.mfc.co.jp/english/se_pharma/sepharma.htm. Accessed September 18, 2012.

32. Becerra N, Nuez LRD, Zanocco AL, Lemp E. Solubilization of dodac small unilamellar vesicles by sucrose esters A fluorescence study. Colloids Surf A Physicochem Eng Asp. 2006;272(1):2-7.

33. BASF. Lutrol L and Lutrol F grades. Available from: http://www. pharma-ingredients.basf.com/Statements/Technical\%20Informations/ EN/Pharma\%20Solutions/03_100102e_Lutrol\%20L\%20and\%20 Lutrol\%20F-Grades.pdf. Accessed July 3, 2012.

34. Cheng CY, Wang JY, Kausik R, Lee KYC, Han S. An ultrasensitive tool exploiting hydration dynamics to decipher weak lipid membranepolymer interactions. J Magn Reson. 2012;215:115-119.

35. Kolter K, Karl M, Nalawade S, Rottmann N. Hot-Melt Extrusion with BASF Pharma Polymers: Extrusion Compendium. 2nd ed. Ludwigshafen: BASF; 2011.
International Journal of Nanomedicine

\section{Publish your work in this journal}

The International Journal of Nanomedicine is an international, peerreviewed journal focusing on the application of nanotechnology in diagnostics, therapeutics, and drug delivery systems throughout the biomedical field. This journal is indexed on PubMed Central, MedLine, CAS, SciSearch ${ }^{\circledR}$, Current Contents ${ }^{\circledR} /$ Clinical Medicine,

\section{Dovepress}

Journal Citation Reports/Science Edition, EMBase, Scopus and the Elsevier Bibliographic databases. The manuscript management system is completely online and includes a very quick and fair peer-review system, which is all easy to use. Visit http://www.dovepress.com/ testimonials.php to read real quotes from published authors. 